\title{
TUBERCULOSE: CARACTERÍSTICAS E PREVALÊNCIA NA POPULAÇÃO PRIVADA DE LIBERDADE DE SISTEMAS DE SAÚDE PRISIONAL DO RIO GRANDE DO SUL - BRASIL
}

\author{
Ana Júlia Reis ${ }^{1}$ \\ Ana Cristina Weber Bavaresco 2 \\ Caroline Busatto \\ Bruna Franke 4 \\ Vanda Hermes ${ }^{5}$ \\ Andreia Rosane de Moura Valim ${ }^{6}$ \\ Suzane Frantz Krug $^{7}$ \\ Lia Gonçalves Possuelo 8
}

\begin{abstract}
RESUMO
No Brasil, a Tuberculose (TB) é um grande problema entre a população privada de liberdade (PPL), sendo a sua magnitude pouco conhecida pela ausência da implantação e segmento de programas de vigilância epidemiológica e controle da TB nesta população. O objetivo deste trabalho foi comparar a prevalência de TB na população geral e entre a PPL nos municípios do estado do Rio Grande do Sul que possuem equipes de saúde prisional implementadas. Realizou-se um estudo ecológico, utilizando dados do Sistema Nacional de Agravos de Notificação (SINAN), no qual avaliou-se o número de casos de TB notificados na população geral e entre a PPL, no período de 2011 a 2013, dos 13 municípios do Rio Grande do Sul (RS) que possuem equipes de saúde prisional. Verificou-se que 7 (53,8\%) municípios estudados ampliaram seus registros de notificação de casos de TB após a implementação das equipes de saúde. Entretanto, ainda são necessárias diversas ações neste sentido, entre elas, maior adesão aos procedimentos para controle da $\mathrm{TB}$, a capacitação dos profissionais de saúde para a identificação de sintomáticos respiratórios, realização de triagem entre ingressos e indivíduos já encarcerados e encaminhamento de exames para o diagnóstico precoce da TB.
\end{abstract}

Palavras-chave: Tuberculose. Penitenciárias. Educação em Saúde.

\begin{abstract}
In Brazil, Tuberculosis (TB) is a big problem among prisoners (PPL), its magnitude being little known by the absence of implementation and maintenance of epidemiological surveillance and TB control programs in this population segment. The objective of this study was to compare the prevalence of TB in the general population and in the PPL between municipalities in the state of Rio Grande do Sul that have health teams working with prisoners. An ecological study was conducted using data from the National Disease

\footnotetext{
${ }^{1}$ Aluna do Curso de Farmácia da Universidade de Santa Cruz do Sul - UNISC. < anajulia_reis@ @otmail.com>

${ }^{2}$ Aluna do Curso de Farmácia da Universidade de Santa Cruz do Sul - UNISC. <ana.cristinaw@ hotmail.com>

${ }^{3}$ Aluna do Curso de Farmácia da Universidade de Santa Cruz do Sul - UNISC. < caroline-busatto@ hotmail.com>

${ }^{4}$ Bolsista Ensino Médio na Universidade de Santa Cruz do Sul - UNISC. <brunafranke@hotmail.com>

${ }^{5}$ Enfermeira Prefeitura Municipal de Santa Cruz do Sul. Programa de Controle da Tuberculose. < $\underline{\text { vanda- }}$ hermes@hotmail.com>.

${ }^{6}$ Docente do Departamento de Biologia e Farmácia. Universidade de Santa Cruz do Sul. 〈avalim@unisc.br〉.

${ }^{7}$ Docente do Departamento de Enfermagem e Odontologia da Universidade de Santa Cruz do Sul - UNISC. <skrug@unisc.br>

${ }^{8}$ Docente do Departamento de Biologia e Farmácia da Universidade de Santa Cruz do Sul - UNISC. <liapossuelo@unisc.br>
} 
Surveillance System (SINAN), in which was assessed the number of TB cases reported in the general population and among the PPL, from 2011 to 2013, in the 13 municipalities of Rio Grande do Sul (RS) that have implanted policies for prisoners' health. It was found that 7 (53.8\%) studied cities expanded their notifications of TB cases after implementation of health teams. Becomes necessary to greater compliance procedures for tuberculosis control, training of health professionals to identify symptomatic patients, conducting screening and ticket between individuals already incarcerated and routing tests for early diagnosis of TB.

Keywords: Tuberculosis. Prisons. Health education.

\section{INTRODUÇÃO}

A função da prisão é proteger a sociedade contra o crime e, para que isto se torne possível, é preciso reduzir as diferenças entre a população carcerária e a sociedade em geral, garantindo-lhes condições dignas de vida e saúde, sendo estas determinantes para as condições físicas e mentais desta população. Geralmente, quando recolhido ao sistema prisional, o cidadão traz problemas de saúde, vícios e até mesmo problemas mentais, que tendem a se agravar com as precárias condições de moradia, alimentação e saúde das penitenciárias (PNSSP, 2010).

O aumento da população encarcerada é um fenômeno que vem sendo observado em inúmeros países, inclusive no Brasil. A disseminação de doenças contagiosas, em especial a infecção pelo HIV/AIDS e tuberculose (TB), constitui sério risco à saúde dos detentos, seus contatos (especialmente familiares e pessoal penitenciário) e para as comunidades nas quais irão se inserir após o livramento. Os estudos sobre a saúde da população prisional brasileira são pouco numerosos, mas todos revelam situação grave no que se refere à infecção pelo HIV e TB, o que confirma a experiência cotidiana dos profissionais de saúde (DIUANA, 2008).

O contexto da saúde penitenciária tem como principais objetivos a prestação de assistência integral, contínua e de boa qualidade às necessidades de saúde de sua população, controlar e reduzir possíveis agravos, implementar ações conforme princípios e diretrizes do SUS, reconhecer a saúde como um direito de cidadania e efetivar o controle social. Assim, verifica-se a importância de ações para o controle e detecção da TB nesta população, com o objetivo de implementá-las com a efetividade de $100 \%$ de cura em pelo menos $85 \%$ dos casos (PNSSP, 2010).

A TB prisional constitui um importante problema de saúde, principalmente em países em desenvolvimento, sendo comuns formas multirresistentes do bacilo devido ao atraso ou falta de diagnóstico da doença, bem como da realização irregular do tratamento. Atualmente, 
a população prisional representa $0,2 \%$ da população do país, no entanto, representa mais de 5\% dos casos de TB notificados anualmente no Sistema Nacional de Agravos de Notificação (SINAN) (BRASIL, 2011; NOGUEIRA; ABRAHÃO; GALESI, 2012; MOREIRA; FÁVELO; MACIEL, 2010).

Entre as ações para o controle da TB preconizados pela Superintendência dos Serviços Penitenciários (SUSEPE) estão a triagem dos indivíduos pela Porta de Entrada e o Rastreamento da TB em Sintomáticos Respiratórios (SUSEPE, 2011). No entanto, a falta de recursos humanos e financeiros, o risco de estigmatização, subvalorização dos sintomas e o pouco acesso a informações são fatores determinantes na dificuldade de detecção dos casos de TB na PPL (BRASIL, 2011; SANCHEZ et al., 2006).

Diversos fatores têm contribuído para o aumento da endemicidade da TB entre populações prisionais, sendo estas relacionadas com condições anteriores ao encarceramento, ou condições desfavoráveis do próprio encarceramento. Dentre estas, destaca-se que os indivíduos geralmente são do sexo masculino, com pouca escolaridade, vindos de comunidades desfavorecidas, usuários de drogas ilícitas, com uma maior prevalência de infecções por HIV, dificuldade de acesso aos serviços de saúde, celas superpopulosas, mal ventiladas e com pouca iluminação e, muitas vezes, até mesmo há falta de informações sobre a doença (BRASIL, 2011, MOREIRA, 2010).

Sendo assim, o presente estudo teve como objetivo verificar a prevalência e as características epidemiológicas da TB na população geral e entre a população privada de liberdade em instituições que possuem equipes de saúde prisional, identificando a proporção desses casos sobre o total de notificações nos municípios onde as instituições carcerárias se localizam, bem como as características das prisões estudadas.

\section{METODOLOGIA}

Realizou-se um estudo ecológico, com a avaliação de dados secundários provenientes do SINAN, onde foram avaliados casos de TB notificados na população geral e na população privada de liberdade (PPL), no período de 2011 a 2013. Foram avaliados dados de 13 municípios do Rio Grande do Sul (RS) que possuem equipes de saúde prisional implementadas. Nestes municípios são totalizadas 20 equipes que atendem 15.998 apenados, com uma cobertura de 53,6\% do total de apenados do estado (Figura 1). Destes municípios, quatro são considerados prioritários para o controle de TB no estado: Santa Cruz do Sul, Porto Alegre, Pelotas e Guaíba (SES/DAS/SEÇÃO SAÚDE PRISIONAL, 2012; RS, 2010). 
A partir da base de dados do SINAN foram obtidos os dados epidemiológicos referentes a cor da pele, sexo e número de casos notificados na população geral e na PPL. A partir dos dados fornecidos pela SUSEPE, avaliou-se a quantidade da PPL destes municípios, número de vagas disponíveis, bem como o déficit de vagas. O cálculo da prevalência foi realizado utilizando-se o número de casos notificados de TB por município, o tipo de entrada todos os casos. A prevalência foi expressa em casos por 100.000 habitantes. Os dados referentes à população geral e PPL foram obtidos no site do Instituto Brasileiro de Geografia e Estatística (IBGE). Todas as bases de dados foram acessadas para atualização dos dados no período de outubro de 2013 a fevereiro de 2014. O cálculo referente à proporção de casos de TB na PPL em relação ao total de casos notificados também foi realizada. Os dados levantados foram tabulados em planilha Excel e os resultados expressos em números absolutos, frequência e prevalência.

Figura 1 - Municípios que possuem equipes de saúde prisional no Estado do Rio Grande do Sul

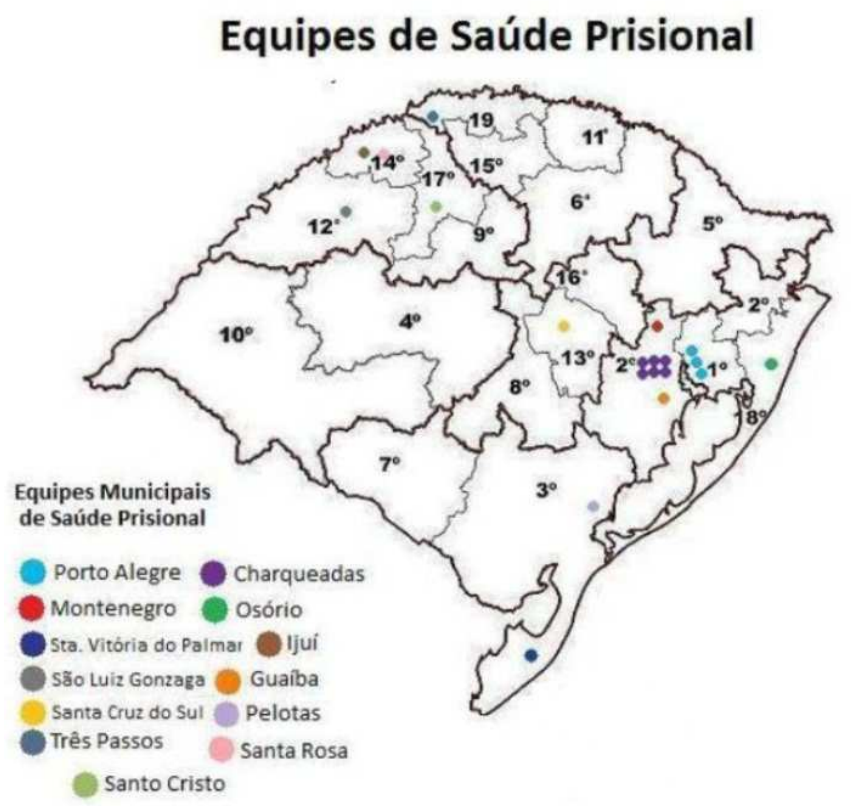

Fonte: SES/DAS/SEÇÃO SAÚDE PRISIONAL/JUL 2012

\section{RESULTADOS E DISCUSSÃO}

Com base nos dados da SUSEPE referente às casas penitenciárias presentes nos 13 municípios avaliados, pode-se verificar uma média de 9.900 vagas disponíveis em presídios, para um total de 13.239 apenados, representando um déficit de vagas de 33,4\%, o equivalente 
a 3.300 apenados a mais que o projetado para estes locais (Tabela 1) (SUSEPE, 2014). Menezes e colaboradores (2002) identificaram um déficit de vagas de $28 \%$, considerando todos os presídios pertencentes ao estado da Bahia (MENEZES, 2002). No presente estudo, o déficit de vagas observado foi de $33,4 \%$, somente entre os municípios estudados, o que corresponde a $53,6 \%$ da cobertura do estado do RS.

Tabela 1 - Déficit de vagas considerando os 13 municípios com equipes de saúde prisional.

\begin{tabular}{lll}
\hline & $\mathbf{n}$ & \% \\
\hline Vagas Disponíveis & 9941 & -- \\
Total de Detentos & 13239 & -- \\
Déficit de Vagas & 3298 & 33,4 \\
\hline
\end{tabular}

No ano de 2013 foram notificados 546 casos de TB na PPL do estado do RS, 513 $(93,9 \%)$ eram do sexo masculino e $336(61,5 \%)$ eram de cor branca (Tabela 2$)$ (DATASUS, 2014). Em estudo realizado por Lima e colaboradores (2012), 97,3\% dos casos de TB foram notificados entre homens. Nogueira e colaboradores (2012) verificaram em seu estudo um total de 50,9\% dos indivíduos com cor da pele branca. Em estudo realizado na penitenciária regional de Santa Cruz do Sul por Pereira e colaboradores (2013), 80,4\% dos indivíduos eram brancos. Em estudo realizado por Rocha e colaboradores (2013), 95\% dos participantes eram homens e $67,5 \%$ eram brancos.

O Brasil apresenta uma das populações mais miscigenadas do mundo, no entanto, o estado do RS tem uma colonização singular devido à presença de imigrantes espanhóis, italianos e alemães, o que explicaria uma maior concentração de indivíduos brancos encontrados no estudo. Além disto, o estado possui indivíduos descendentes de africanos e ameríndios, mistura esta exclusiva do sul do Brasil (CALLEGARI-JACQUES, 2003; MARRERO, 2007; GUERREIRO-JUNIOR, 2009). Estudo realizado por Moreira e colaboradores (2010), no estado do Espírito Santo, encontrou uma frequência de 21,9\% dos indivíduos de cor branca entre a PPL e de $32,3 \%$ entre a população em geral, o que reforça as diferenças étnicas observadas no estado do Rio Grande do Sul. 
Tabela 2 - Características epidemiológicas entre casos de TB na PPL em 2013

\begin{tabular}{lll}
\hline Variável & $\mathbf{N}$ & $\mathbf{\%}$ \\
\hline Sexo & & \\
Masculino & 513 & 93,9 \\
Feminino & 33 & 6,1 \\
Cor da Pele & & \\
Branco & 336 & 61,5 \\
Não Branco & 210 & 38,5 \\
Total & $\mathbf{5 4 6}$ & $\mathbf{1 0 0}$ \\
\hline
\end{tabular}

A média de prevalência de TB na população geral nos 13 municípios estudados foi de 77,7 casos/100.000 habitantes, conforme apresentado na Tabela 3 (SINAN, 2014; IBGE, 2014). Lemos et al. (2009) encontraram uma média de 38 casos/100.000 habitantes, inferior à encontrada neste estudo. As prevalências apontadas nos estudos são inferiores às observadas no presente estudo, possivelmente, devido ao fato de termos incluído na população em questão quatro municípios prioritários para o controle da TB no RS e também por este ser um dos estados brasileiros com maior prevalência de TB do Brasil (HIJJAR, 2006; SINAN, 2014).

Tabela 3 - Prevalência de TB na população geral dos municípios do RS estudados (casos/100.000 habitantes)

\begin{tabular}{llll}
\hline Município & População & Casos em 2013 & $\mathbf{N}^{\mathbf{0}}$ de casos/100.000 hab. \\
\hline Porto Alegre & 1.467 .816 & 2.704 & 184 \\
Montenegro & 62.484 & 40 & 64 \\
Santa Vitória do Palmar & 31.618 & 5 & 16 \\
São Luiz Gonzaga & 35.344 & 6 & 17 \\
Santa Cruz do Sul & 124.577 & 92 & 74 \\
Três Passos & 24.665 & 7 & 28 \\
Santo Cristo & 12.778 & 1 & 8 \\
Santa Rosa & 71.665 & 7 & 10 \\
Charqueadas & 37.589 & 129 & 343 \\
Osório & 43.256 & 34 & 79 \\
Ijuí & 82.276 & 22 & 27 \\
Guaíba & 98.688 & 94 & 95 \\
Pelotas & 341.180 & 223 & 65 \\
\hline Média & & & $\mathbf{7 7 , 7}$ \\
\hline
\end{tabular}

Considerando a prevalência de casos entre a PPL dos treze municípios do RS em questão, obteve-se uma média de 1.995 casos/100.000 habitantes, sendo que nos municípios de Porto Alegre, Santa Cruz do Sul e Charqueadas foram observadas as taxas mais elevadas, como apresentado na Tabela 4. Em estudo realizado no ano de 2011, Pereira e colaboradores (2013) observaram no presídio regional de Santa Cruz do Sul uma prevalência de 1898 casos/100.000 habitantes, valor este semelhante ao encontrado no presente estudo. Rocha e colaboradores (2013), em estudo realizado em uma penitenciária do estado do Rio Grande do 
Sul, obtiveram uma prevalência de 9.542 casos/100.000 habitantes. Em estudo realizado com a PPL da Etiópia, por Abebe e colaboradores (2011) encontraram uma prevalência de 1.913 casos/100.000 habitantes. No estado de São Paulo, a prevalência encontrada foi de 787 casos/100.000 (ABRAHÃO, 2006), indicando que os resultados obtidos no presente estudo são semelhantes àqueles observados em outros locais e, principalmente, considerando a população em geral, são índices extremamente elevados.

Tabela 4 - Prevalência de TB na PPL, 2013

\begin{tabular}{llll}
\hline Município & PPL & Casos em 2013 & Casos/100.000 hab. \\
\hline Porto Alegre & 5404 & 345 & 6384 \\
Montenegro & 458 & 9 & 1965 \\
Santa Vitória do Palmar & 73 & 0 & 0 \\
São Luiz Gonzaga & 147 & 0 & 0 \\
Santa Cruz do Sul & 456 & 32 & 7017 \\
Três Passos & 206 & 0 & 0 \\
Santo Cristo & 58 & 0 & 0 \\
Santa Rosa & 381 & 4 & 1049 \\
Charqueadas & 3171 & 107 & 3374 \\
Osório & 1133 & 17 & 1500 \\
Ijuí & 629 & 3 & 476 \\
Guaíba & 247 & 3 & 1214 \\
Pelotas & 876 & 26 & 2968 \\
\hline Média & & & $\mathbf{1 9 9 5}$ \\
\hline
\end{tabular}

A proporção de casos de TB na PPL em relação ao total de casos notificados nos municípios estudados variou de 0 a 82,9\%. Verificou-se que 7 (53,8\%) municípios apresentaram uma maior proporção de casos de TB nas penitenciárias no ano de 2013, quando comparados aos períodos anteriores, como pode ser visto na Tabela 5. A inserção da variável institucionalizado (presídio) no SINAN ocorreu no ano de 2007 e desde lá é possível identificar a proporção de casos de TB entre apenados. No ano de 2012 esta proporção variou de $1,5 \%$ a $18 \%$ nas unidades federativas, com uma média de $7,2 \%$. No presente estudo a proporção média variou de $18,7 \%$ a $22,2 \%$ no período estudado, valor este que está acima da média nacional. Estes dados demonstram que a recente implantação das equipes de saúde prisional no RS tem melhorado a capacidade diagnóstica dos casos de TB na PPL, entretanto, ainda é necessário melhorar e ampliar a capacitação das equipes de saúde prisional para seguimento das ações e estratégias para o controle da tuberculose nesta população vulnerável. 
Tabela 5 - Número de casos notificados na população geral, número de casos na PPL e proporção de casos de TB na população prisional entre o total de casos notificados, 2011 a 2013

\begin{tabular}{llllllllll}
\hline & $\mathbf{2 0 1 1}$ & \multicolumn{3}{c}{$\mathbf{2 0 1 2}$} & \multicolumn{3}{c}{$\mathbf{2 0 1 3}$} \\
\hline Município & Geral & PPL & $\mathbf{\%}$ & Geral & PPL & \% & Geral & PPL & \% \\
\hline Porto Alegre & 2.611 & 323 & 12,44 & 2.637 & 282 & 10,7 & 2.704 & 345 & 12,7 \\
Montenegro & 52 & 26 & 50,0 & 42 & 18 & 42,8 & 40 & 9 & 22,5 \\
Santa Vitória do Palmar & 13 & 1 & 7,7 & 5 & 0 & 0,0 & 5 & 0 & 0,0 \\
São Luiz Gonzaga & 11 & 0 & 0,0 & 8 & 3 & 37,5 & 6 & 0 & 0,0 \\
Santa Cruz do Sul & 68 & 6 & 8,8 & 60 & 14 & 23,3 & 92 & 32 & 34,8 \\
Três Passos & 13 & 0 & 0,0 & 4 & 0 & 0,0 & 7 & 0 & 0,0 \\
Santo Cristo & 1 & 0 & 0,0 & 0 & 0 & 0,0 & 1 & 0 & 0,0 \\
Santa Rosa & 14 & 2 & 14,3 & 9 & 0 & 0,0 & 7 & 4 & 57,1 \\
Charqueadas & 152 & 123 & 80,9 & 113 & 85 & 75,2 & 129 & 107 & 82,9 \\
Osório & 34 & 21 & 61,8 & 46 & 27 & 58,7 & 34 & 17 & 50,0 \\
Ijuí & 17 & 1 & 5,9 & 13 & 1 & 7,7 & 22 & 3 & 13,6 \\
Guaíba & 55 & 1 & 1,8 & 64 & 3 & 4,7 & 94 & 3 & 3,2 \\
Pelotas & 213 & 10 & 4,7 & 254 & 29 & 11,4 & 223 & 26 & 11,6 \\
\hline Média & & & $\mathbf{1 8 , 7}$ & & & $\mathbf{2 0 , 9}$ & & & $\mathbf{2 2 , 2}$ \\
\hline
\end{tabular}

\section{CONCLUSÃO}

Os resultados apresentam a prevalência de tuberculose entre a PPL extremamente elevada quando comparada com a população em geral e a PPL de outros estudos, havendo uma predominância de indivíduos com cor da pele branca na população estudada.

A TB nos presídios constitui-se num sério problema de saúde pública, pois afeta, além de apenados, familiares e funcionários, durante e após a detenção. Apesar da implementação das equipes de saúde prisional, a realização das ações de prevenção à TB parecem ainda não ocorrer em plena consonância com as ações recomendadas pelo Programa Nacional de Controle da Tuberculose, bem como pelo Plano Nacional de Saúde no Sistema Prisional. Torna-se necessária a maior adesão aos procedimentos para controle da tuberculose, a capacitação dos profissionais de saúde para a identificação de sintomáticos respiratórios, com a consequente realização de triagem destes, para diagnóstico precoce da TB. Neste contexto, o estabelecimento de rotinas e fluxos para exames diagnósticos devem ser definidos no sistema prisional, para que o rastreamento permita dimensionar o problema e sensibilizar autoridades e comunidade carcerária. 


\section{REFERÊNCIAS}

ABRAHÃO, R. M. C. M.; NOGUEIRA, P. A.; MALUCELLI, M. I. C. Tuberculosis in county jail prisoners in the western sector of the city of São Paulo, Brazil. . Int J Tuberc Lung Dis, v. 10, n. 2 p. 203-208, 2006.

ADEBE, D. S., et al. Prevalence of pulmonary tuberculosis and associated risk factors in Eastern Ethiopian prisons. Int J Tuberc Lung Dis, v. 15, n. 5 p. 668-673, 2011.

BRASIL. Manual de Recomendações para o Controle da Tuberculose no Brasil. Brasília: Ministério da Saúde, 2011.

CALLEGARI-JACQUES, S. M., et al. Historical genetics: Spatiotemporal analysis of the formation of the Brazilian population. Am. J. Hum. Biol, v. 15 p. 824-834, 2003.

DIUANA, V., et al. Saúde em prisões: representações e práticas dos agentes de segurança penitenciária no Rio de Janeiro, Brasil. Cad. Saúde Pública. Rio de Janeiro, v. 24, n. 8 p. 1887-1896, 2008.

GUERREIRO-JUNIOR, V., et al. Genetic Signatures of parental contribution in black and white populations in Brazil. Genetics and Molecular Biology, v. 32, n. 1 p. 1-11, 2009.

HIJJAR, M. A.; PROCÓPIO, M. J. Tuberculose - Epidemiologia e Controle no Brasil. Revista do Hospital Universitário Pedro Ernesto. Rio de Janeiro, v. 5, 2006.

IBGE. Instituto Brasileiro de Geografia e Estatística. Disponível em: <http://www.cidades.ibge.gov.br/xtras/home.php>. Acesso em: 10 fev. 2014.

LEMOS, A. C. M.; MATOS, E. D.; BITTENCOURT, C. N. Prevalência de TB ativa e TB latente em internos de um hospital penal na Bahia. J Bras Pneumol, v. 35, n. 1 p. 63-68, 2009.

LIMA, R. C. M.; JUNIOR, A. O. C.; SANTOS, L. C. P. Tuberculose no Complexo Prisional de Aparecida de Goiânia - GO. Ensaios e Ciência: Ciências Biológicas, Agrárias e da Saúde, v. 16, n. 1 p. 43-53, 2012.

MARRERO, A. R., et al. Pre-and post-Columbian gene and cultural continuity: the case of the Gaucho from southern Brazil. Hum Hered, v. 64, n. 3 p. 160-171, 2007.

MENEZES, R. P. O. Projeto de implantação do controle da Tuberculose nas instituições penais do município de salvador/BA. Boletim de Pneumologia Sanitária, v. 10, n. 2, 2002.

MOREIRA, T. R.; FÁVERO, J. L.; MACIEL, E. L. N. Tuberculose no sistema prisional Capixaba. Revista Brasileira de Pesquisa em Saúde, v. 12, n. 1 p. 26-33, 2010.

NOGUEIRA, P. A.; ABRAHÃO, R. M. C. M.; GALESI, V. M. N. Tuberculosis and latent tuberculosis in prison inmates. Rev Saúde Pública, v.46, n. 1 p. 119-27, 2012.

PEREIRA, C. C.; et al. Prevalência de Sintomáticos Respiratórios e Tuberculose Ativa em uma penitenciária do Sul do Brasil. Rev Epidemiol Control Infect, v. 3, n. 3 p. 99-104, 2013. 
PNSSP. Plano Nacional de Saúde no Sistema Penitenciário. Brasília: Ministério da Saúde, 2010.

ROCHA, J. Z., et al. Respiratory symptoms and active tuberculosis in a prison in southern Brazil: Associated epidemiologic variables. Rev Epidemiol Control Infect, v. 4, 2013.

RS. Plano estadual de controle da Tuberculose, 2010. Disponível em: <http://www.saude.rs.gov.br/upload/1339784445_Plano\%20Estadual\%20de\%20Controle\%20 da\%20Tuberculose.pdf>. Acesso em: 3 fev. 2014.

SÁNCHEZ, A. R. et al. A tuberculose nas prisões: uma fatalidade?. Cad Saúde Pública, v. 22, n. 12 p. 2510, 2006.

SES/DAS/SEÇÃO SAÚDE PRISIONAL. Seção de Saúde Prisional: Rio Grande do Sul, 2012.

SINAN, RS. Sistema de Informação de Agravos de Notificação. "Tuberculose: Casos confirmados notificados no Sistema de Informação de Agravos de Notificação, SinanNet”. Disponível em: <http://dtr2004.saude.gov.br/sinanweb/tabnet/dh?sinannet/tuberculose/bases/ tubercbrnet.def>. Acesso em: 23 fev. 2014.

SUSEPE. Política de atenção integral à saúde prisional, 2011. Disponível em: <http://www.susepe.rs.gov.br/upload/1315597369_SAUDE.pdf>. Acesso em: 25 fev. 2014.

SUSEPE. Superintendência dos Serviços Penitenciários: Presídios e Delegacias Penitenciárias, 2013. Disponível em: 〈http://www.susepe.rs.gov.br/capa.php>. Acesso em: 14 fev. 2014. 